\title{
The Impact of Sustained Metformin use on Survival in Diabetes Patients of Operable Colorectal Cancer: A Nationwide Cohort Study
}

Ping-Teng Chu ( $\sim$ kazuya.paper@gmail.com )

Kaohsiung Veterans General Hospital https://orcid.org/0000-0001-6337-8199

Tzu-Jung Chuang

National Cheng Kung University

Tung-Ho Wu

Kaohsiung Veterans General Hospital

Wei-Chun Huang

Kaohsiung Veterans General Hospital

Jui-Ho Wang

Kaohsiung Veterans General Hospital

Research Article

Keywords: metformin, colorectal cancer, diabetes

Posted Date: October 25th, 2021

DOI: https://doi.org/10.21203/rs.3.rs-994038/v1

License: (9) This work is licensed under a Creative Commons Attribution 4.0 International License.

Read Full License 


\section{Abstract}

\section{Background}

Colorectal cancer is one of the most common cancers in the Western world and in Taiwan and the diabetes has been proven as an independent risk factor for incidence. Metformin, the drug of choice for the therapy of type 2 diabetes, has been shown to reduce the risk of developing colorectal cancer. Our study was investigated the impact of continuous use of metformin on the survival of diabetic patients after surgery for colorectal cancer.

\section{Methods}

This study identified 12,512 patients with colorectal cancer and type 2 diabetes who underwent curative surgery between 2000 and 2012 were collected from the Taiwanese population-based National Health Insurance Research Database. A total of 6,222 patients were included in the matched cohort, to adjust for differences in baseline variables. Time-dependent covariates in the cox proportional hazard model was used to examine the impacts of metformin use on survival.

\section{Results}

The average duration of follow-up was 49.4 months in the Metformin use group and 53.7 months in the non-Metformin use group. The Cox proportional hazard model revealed that continuous metformin use post operation was associated with 5-year survival benefit (HR: $0.23,95 \% \mathrm{Cl}: 0.20-0.26)$. Furthermore, continuous metformin use also showed a benefit of decreasing liver metastases (HR: $0.83,95 \% \mathrm{Cl}$ : 0.71 0.97).

\section{Conclusions}

Sustained Metformin use is associated with a significant survival benefit in diabetic colorectal cancer patients after operation, suggesting a potential anti-tumorigenic effect. Moreover, there was also a benefit of decreasing liver metastases in patients who received continuous metformin use.

\section{Introduction}

Even though advancements in healthcare has contributed to the improved diagnosis and treatment of disease, colorectal cancer is still the third most common cancer in terms of incidence and ranks second in terms of mortality worldwide, with 1.8 million new colorectal cancer cases and 881,000 deaths occurring in 2018 worldwide [1]. In Taiwan, colorectal cancer is the most common cancer and the third leading cause of cancer, which claimed 24.7 lives per 100,000 population in 2015 [1]. The most common pathological type of colorectal cancer is adenocarcinoma (>95\%) and the remaining rare types include carcinoid tumor, lymphoma and sarcoma [2]. Approximately 80 percent of colorectal cancers are localized to the colorectal wall and/or regional lymph nodes, where the only curative treatment would be operable resection, which means the primary target of surgical resection for invasive colorectal cancer is 
to completely remove the cancer, including the major vascular pedicles and lymphatic drainage of the involved colorectal segment [3].

The worldwide incidence and prevalence of type II diabetes mellitus has increased by 4 times over the period of 1980 and 2004 and its prevalence is still growing. The population of diabetic patients is expected to rise from 415 million in 2015 to 642 million by 2040 [4, 5]. In Taiwan, diabetes mellitus is one of the top ten leading causes of death. Diabetes mellitus and colorectal cancer both involve various common risk factors, including obesity, inactivity and western diet. However, diabetes mellitus is regarded as an independent risk factor for developing colorectal cancer [6, 7]. The risk of developing colon cancer among diabetic patients is approximately 38 percent higher than non-diabetic persons and the risk of having rectal cancer among diabetics is 20 percent greater than non-diabetic individuals [8].

Metformin is a biguanide that works as a medication treatment for type II diabetes mellitus. Many studies have revealed that metformin may help lower the risk of developing certain cancers, including colorectal cancer [9-11]. In addition, the studies indicate that the antineoplastic effect of metformin can resolve the inhibition of the mammalian target of the rapamycin (mTOR) pathway and can prevent cancer cells from growing $[12,13]$. In this regard, using metformin may potential contribute to the primary prevention and treatment of colorectal cancer as an adjuvant therapy [13-15]. Therefore, we conducted a nationwide population-based retrospective cohort study using the Taiwan National Health Insurance (NHI) database which aims to investigate the impact of the continuous usage of metformin treatment in diabetic patients with operable colorectal cancer.

\section{Materials And Methods}

This longitudinal cohort study was conducted using a Taiwanese population-based National Health Insurance Research Database (NHIRD). The NHI is a single-payer system and has been compulsory for the health insurance program of Taiwan since 1995. The NHI covers $>99 \%$ of Taiwan's 23 million population. As all the identifiable information had been encrypted prior to the release of the Taiwanese NHIRD, it was not necessary to obtain informed consent for this study.

\section{Study cohort}

The patients with a primary diagnosis of colorectal cancer (ICD-9-CM code: 153-154) combined with diabetes mellitus (ICD-9-CM code: A181, 250) using the registry for catastrophic illness patients (HV) files during January 2000 to December 2012 were included. The inclusion criteria are: (1) Diagnosis of diabetes mellitus before index date (2) received oral diabetes medications $\geq 28$ days within 1 year before the index date. In addition, during the follow-up period, if the interval between two doses of metformin is within 14 days, it was considered to be taken continuously.

Patients aged $<18$ years with unspecified malignant neoplasm of the anus (ICD-9-CM, 154.3) were excluded. Patients who underwent curative surgery were surgery 60 days before the diagnosis to half a year after the diagnosis enrolled and the time of surgery was used as an index date $(N=91,649)$. To focus 
on acquired colorectal cancer, we excluded patients with a follow-up period of less than 3 months, liver metastases before the index date, and missing data $(N=12,512)$. The cohort period is $2000-2012$, and it is flowing up to 2013.

\section{Matched cohort}

A total of 12,512 patients were enrolled in this study, including 9,401 metformin users. Propensity score matched pair sampling (1:1 ratio) was used to adjust for differences in baseline characteristics. Our matching conditions include age, gender, year of surgery, income, Charlson comborbidity Index (CCl), adapted Diabetes Complications Severity Index (aDCSI), other non-metformin diabetes drugs, statin, radiotherapy, chemotherapy, etc. After the two groups are adjusted, the comparability can be increased, and be performed to identify the risk of survival to patients. We compared the distribution of demographic factors and clinical characteristics between the study cohort (i.e., metformin use) and the matched control cohort (i.e., non-metformin use). The standardized mean difference (SMD) of covariates was set to 0.1 by comparing the study cohort and control cohort before and after matching. Finally, 6,222 patients were included in the matched cohort (Fig. 1)

\section{Primary study outcome}

The primary study outcome was the overall survival (OS) after 5 years, and the secondary outcome was liver metastasis of disease-free survival (DFS), determined from the NHIRD. OS was calculated as the time interval between the index date and the date of death from colorectal cancer; whereas, DFS was the time from surgery to liver metastasis or death.

\section{Statistical analyses}

Descriptive statistics were calculated for all variables, with categorical data shown as percentile values, and continuous variables as means and standard deviations. Continuous variables were analyzed using paired $t$ tests and categorical variables were evaluated by Chi-squared tests. According to the characteristics of the data, we used time-dependent covariates in the cox proportional hazard model were constructed with accompanying $95 \% \mathrm{Cls}$ and hazard ratios after adjusting for age, sex, income, Charlson Comorbidity Index (CCl) and colorectal cancer treatment, to examine the impact of metformin use on survival. We use the cox proportional hazard model analysis of time-varying covariates (also called timevarying covariates). And use time correlation or change over time to indicate that the independent variable (risk factor) may change over time. In our study, the survival time of patients after using metformin may be significantly related. During the study follow-up, the use of metformin will change (the time interval for each person to use metformin is different). The traditional cox proportional hazard model analysis only judges whether to adopt metformin, which is a fixed time mode.

We used Kaplan Meyer analysis to compare the survival differences between the two ethnic groups. Used the log-rank test to define the association between the two groups and survival (Fig. 4 \& Fig. 5). 
Data management and analyses were conducted using SAS statistical software, version 9.4. A P $<0.05$ was considered statistically significant.

\section{Results}

For comparisons between the metformin use and non-metformin use groups in unmatched analysis, the average follow-up duration was $39.89 \pm 18.52$ and $40.87 \pm 19.30$ months, and the duration from index day to recurrence was $49.37 \pm 36.80$ and $53.72 \pm 40.35$ months for the metformin and non-metformin group respectively. After 1:1 propensity score matching, the average follow-up duration was $42.15 \pm 18.93$ and $40.87 \pm 19.30$ months and the duration from index day to recurrence was $55.73 \pm 40.78$ and $53.72 \pm 40.35$ months in metformin and non-metformin group respectively.

Table 1 summarizes the distribution of the baseline demographic characteristics and medication use of unmatched patients. The mean age $( \pm S D)$ of the metformin and non-metformin cohorts was $68.49 \pm 9.75$ and $70.03 \pm 10.17$ years, respectively. The non-metformin cohort was markedly older than the metformin cohort. 
Table 1

Demographic and clinical characteristics in unmatched analysis

\begin{tabular}{|c|c|c|c|c|}
\hline & $\begin{array}{l}\text { Metformin use } \\
\mathrm{N}=9,401\end{array}$ & $\begin{array}{l}\text { Non metformin use } \\
\mathrm{N}=3,111\end{array}$ & $p$ value & SMD \\
\hline Age & $68.49 \pm 9.75$ & $70.03 \pm 10.17$ & $<.0001$ & -0.155 \\
\hline Gender & & & 0.5835 & \\
\hline Male & $5199(55.30)$ & 1738(55.87) & & -0.011 \\
\hline Female & $4202(44.70)$ & 1373(44.13) & & 0.011 \\
\hline Income (NTD/month) & & & $<.0001$ & \\
\hline$<20,000$ & $3997(42.52)$ & $1541(49.53)$ & & -0.141 \\
\hline $20,000-39,999$ & $3748(39.87)$ & 1045(33.59) & & 0.131 \\
\hline$\geq 40,000$ & 1656(17.62) & $525(16.88)$ & & 0.020 \\
\hline $\mathrm{CCl}$ & & & $<.0001$ & \\
\hline$<3$ & $8430(89.67)$ & 2608(83.83) & & 0.173 \\
\hline$\geq 3$ & $971(10.33)$ & $503(16.17)$ & & -0.173 \\
\hline aDCSI & & & 0.0778 & \\
\hline 0 & $1359(14.46)$ & $490(15.75)$ & & -0.036 \\
\hline$\geq 1$ & $8042(85.54)$ & $2621(84.25)$ & & 0.036 \\
\hline \multicolumn{5}{|l|}{ Diabetes medication } \\
\hline Sulfonylurea & $7077(75.28)$ & $2526(81.20)$ & $<.0001$ & -0.144 \\
\hline a-glucosidase inhibitors & $1361(14.48)$ & $373(11.99)$ & 0.0005 & 0.073 \\
\hline Glinide & 154(1.64) & $52(1.67)$ & 0.8991 & -0.003 \\
\hline Insulin & $1282(13.64)$ & $663(21.31)$ & $<.0001$ & -0.203 \\
\hline Thiazolidinediones & $1373(14.60)$ & $261(8.39)$ & $<.0001$ & 0.196 \\
\hline Statin & $3003(31.94)$ & $753(24.20)$ & $<.0001$ & 0.173 \\
\hline \multicolumn{5}{|l|}{ Cancer treatment } \\
\hline Radiotherapy & 1664(17.70) & $535(17.20)$ & 0.5227 & 0.013 \\
\hline Chemotherapy & $5989(63.71)$ & 1922(61.78) & 0.0536 & 0.040 \\
\hline
\end{tabular}


As shown in Table 2, more patients in the Metformin use group was noted. After 1:1 propensity score matching, the remaining 6,222 were divided for comparison into two balanced cohorts, including 3,111 in the Metformin use group and 3,111 in the non-Metformin use group. There were no significant differences in the demographic or treatment characteristics between the two groups which was confirmed by standardized mean difference. 
Table 2

Demographic and clinical characteristics after matching

\begin{tabular}{|c|c|c|c|c|}
\hline & $\begin{array}{l}\text { Metformin use } \\
N=3,111\end{array}$ & $\begin{array}{l}\text { Non metformin use } \\
\mathrm{N}=3,111\end{array}$ & $p$ value & SMD \\
\hline Age & $69.88 \pm 9.54$ & $70.03 \pm 10.17$ & 0.5419 & -0.015 \\
\hline Gender & & & 0.9186 & \\
\hline Male & 1742(55.99) & 1738(55.87) & & 0.003 \\
\hline Female & $1369(44.01)$ & 1373(44.13) & & -0.003 \\
\hline Income (NTD/month) & & & 0.9191 & \\
\hline$<20,000$ & $1550(49.82)$ & $1541(49.53)$ & & 0.006 \\
\hline $20,000-39,999$ & 1030(33.11) & 1045(33.59) & & -0.010 \\
\hline$\geq 40,000$ & $531(17.07)$ & $525(16.88)$ & & 0.057 \\
\hline $\mathrm{CCl}$ & & & 0.2351 & \\
\hline$<3$ & 2642(84.92) & 2608(83.83) & & 0.030 \\
\hline$\geq 3$ & $469(15.08)$ & $503(16.17)$ & & -0.030 \\
\hline aDCSI & & & 0.3891 & \\
\hline 0 & $515(16.55)$ & $490(15.75)$ & & 0.022 \\
\hline$\geq 1$ & 2596(83.45) & $2621(84.25)$ & & -0.022 \\
\hline \multicolumn{5}{|l|}{ Diabetes medication } \\
\hline Sulfonylurea & 2564(82.42) & 2526(81.20) & 0.2118 & 0.032 \\
\hline a-glucosidase inhibitors & $380(12.21)$ & $373(11.99)$ & 0.7856 & 0.007 \\
\hline Glinide & $56(1.80)$ & $52(1.67)$ & 0.6978 & 0.010 \\
\hline Insulin & $634(20.38)$ & $663(21.31)$ & 0.3654 & -0.023 \\
\hline Thiazolidinediones & $262(8.42)$ & 261(8.39) & 0.9636 & 0.001 \\
\hline Statin & $748(24.04)$ & $753(24.20)$ & 0.8822 & -0.004 \\
\hline \multicolumn{5}{|l|}{ Cancer treatment } \\
\hline Radiotherapy & $524(16.84)$ & $535(17.20)$ & 0.7106 & -0.018 \\
\hline Chemotherapy & 1949(62.65) & 1922(61.78) & 0.4802 & 0.009 \\
\hline
\end{tabular}


The 5-year overall survival consistently favored the Metformin use group across the majority of prespecified subgroups. The Cox proportional hazard model with time-dependent covariates showed that the continuous use of metformin was associated with a 5-year survival benefit (HR: $0.23,95 \% \mathrm{Cl}: 0.20$ $0.26, p<.0001$ ) after 1:1 propensity score matching (Figure 2). As shown in Figure 3 , a benefit of decreased liver metastases (HR: $0.83,95 \% \mathrm{Cl}: 0.71-0.97, p=.0169)$ was shown in the group with continuous metformin use. In addition, according to the Kaplan Meyer curve, the 5-year survival rate of the two groups was significantly improved (Figure 4), but liver metastasis was not significant (Figure 5).

\section{Discussions}

In post-operative colorectal cancer patients with diabetic mellitus, continuous use of metformin was associated with a significant survival benefit and a further reduction in liver metastases, implying a potential anti-tumorigenic effect of metformin.

In general, people with diabetes have been associated with inferior survival outcomes and are also at increased risk of developing colorectal cancer [16-18]. This public health concern has been raised globally in response to the high prevalence of type II diabetes mellitus in patients with colorectal carcinoma, while the prognosis of patients with colorectal carcinoma is also significantly affected by their high blood sugar levels [19-22]. The risk of colorectal carcinoma for people with diabetes mellitus has been mentioned in some emerging researches, which suggest that diabetic patients with inadequate glycemic control may be at an even higher risk of colorectal carcinoma and often receive polytherapy [23]. Hyperinsulinemia, hyperglycemia and chronic inflammation may cause a higher risk of colorectal cancer [24-27]. Patients with diabetic colorectal cancer have been shown to have shorter overall survival $[7,17,26]$.

Metformin is the most extensively prescribed first-line orally administered drug for type II diabetes mellitus $[13,28]$. With the amount of metformin use nearly doubling over the past ten years, singleingredient metformin has been prescribed as non-insulin anti-diabetic treatment for approximately one in every two patients [28]. The American Diabetes Association indicated that metformin monotherapy would be the most appropriate therapy in the initial stage of the disease.

Some studies on antidiabetic medication for alleviating cancer have been conducted due to the fact that hyperinsulinemia, associated with metabolic syndromes or type II diabetes mellitus, is linked to increased colorectal cancer risk [29]. The potential anticancer effects of metformin derive from its multiple activities, and thus the reduced cancer occurrence and death rate in diabetic patients, as well as in patients with colorectal cancer, have been linked to the use of metformin [30]. The chemoprevention properties delivered by metformin inhibit the transformative and hyperproliferative processes that trigger cancer-causing events [13]. Even though research on the molecular mechanisms by which metformin influences numerous cancers is still lacking, suppression of mechanistic target of rapamycin (mTOR) signaling by triggering ataxia telangiectasia mutated (ATM) and liver kinase B1(LKB1) and then adenosine monophosphate-activated protein kinase (AMPK)-dependent and AMPK-independent 
pathways, along with energy metabolism aberration, cell cycle arrest, and apoptosis or autophagy induction, have appeared as key inhibitors in these processes to prevent protein synthesis and cells from growing [14]. Metformin is able to trigger p53 by activating AMPK and hence eventually put a stop to the cell cycle. Therefore, in consideration of metformin's prospects on cancer treatment, metformin cannot only be applied in radiotherapy and chemotherapy but also be utilized to prevent many types of cancers [31]. There is a hypothesis about synergistic or additive inhibitory effect of metformin with acetylsalicylic acid (ASA, aspirin) which a drug prescribed for cardioprotection of patient with or without diabetes mellitus II and also inhibit the mTOR signalling pathway. However, randomized controlled trials are required to verify this hypothesis [38].

The use of metformin can reduce the risk and mortality of cancer among diabetic patients. Evans et al indicated that cancer incidence for patients who received metformin was lower than those without metformin. Recent studies also revealed that the risk of rectal cancer decreased, even with a small amount of metformin, which prevents the growth of cancer cells and reduces their expansion. Recurrencefree survival and cancer specific survival indicators also improved through metformin treatment in patients with colorectal cancer [32]. According to Hosono et al a similar effect was also found in the proliferation of cancer cells in colon cancer, as small doses of metformin (250 mg/day) were associated with the destruction of aberrant crypt foci (ACE), which is an indicator related to the presence of colon cancer [33]. High-doses of metformin were shown to reduce mortality in patients with colorectal cancer [34]. In addition, according to Garrett's results, the mortality of patients with colorectal cancer, ranging from grade one to four, was reduced by $40 \%$ due to the use of metformin [35]. Studies have shown that there is no association between diabetes or metformin treatment and recurrence-free or disease-free survival after surgery for colorectal cancer [39]. In difference our results, it is speculated that differences in race may cause such results. In this study, the statistics also echoed the above-mentioned effects, in which the continuous use of metformin proved to reduce the risk of colorectal cancer in operable patients. Furthermore, the use of metformin was shown to be beneficial for the patients in terms of overall 5-year survival. More importantly, the continuous use of metformin also showed benefits for decreasing liver metastases, which has never been discussed in existing published literature.

The effectiveness of metformin in cancer treatment may be affected by four non-identical factors, namely hypoxic cancer cells, the number of cancer stem cells, cell's inherent sensitivity and the rate of cancer cells' growth and spreading (36). Metformin can be applied as a complementary therapeutic medium to cure cancer as it is able to lower the probability of cancer occurrence, decrease the mortality rate caused by cancers, enhance the reaction to therapy in cancer cells during radiotherapy and chemotherapy, alleviate tumor activity and malignity, decrease the possibility of recurrence and lower the detrimental impacts caused by androgen deprivation therapy. Furthermore, metformin can be used to prevent cancers and improve the therapies of cancers and non-malignant tumors. However, more comprehensive research and investigation on metformin's antitumor effects on patients without diabetes is required; and further detailed information on various cells and radiotherapy is needed from the aspects of biology and genetics [31]. 
However, a few constraints in this study should be disclosed. Firstly, the NHI database does not provide access to some recognized potentially key clinical covariates, including diet type, smoking, and alcohol use. Secondly, timewise biases were unavoidable as is the case with many experimental researches on this topic [37].

\section{Conclusion}

In conclusion, metformin use in operable patients with type II diabetes mellitus was associated with a decreased risk of colorectal carcinoma in this nationwide cohort study [38]. In addition, continuous metformin use can also prevent liver metastases, which may help dispel the concept that metformin only benefits the decrease of blood glucose and gives credit to its auxiliary role in cancer treatment. Recent reports have demonstrated inhibition of epithelial to mesenchymal (EMT) genes and specific targeting of stem cells by metformin, thus supporting its potential role in fighting cancer metastases [36]. Further randomized research is needed to investigate the actual role of metformin in the treatment of colorectal patients with diabetes mellitus.

\section{Declarations}

\section{Ethics approval and consent to participate}

This study was approved by the Institutional Review Board of Kaohsiung Veterans General Hospital (VGHKS15-EM10-02). For this type of study, formal consent is not required.

\section{Consent for publication}

Not applicable. This study used secondary data.

\section{Availability of data and materials}

Researchers must follow the Computer-Processed. Personal Data Protection Law and related regulations in Taiwan, so the data used in this study could not be taken out of Health and Welfare Data Center (HWDC).

\section{Competing interests}

All authors have no conflict of interest.

\section{Funding}




\section{Authors' contributions}

Ping-Teng Chu, Jui-Ho Wang, and Tzu-Jung Chuang devised the main conceptual ideas, and Methodology. Tzu-Jung Chuang performed the analysis, and data curation. Tung-Ho Wu assisted with Resources. Wei-Chun Huang, and Jui-Ho Wang proposed the clinical experiment in discussions with PingTeng Chu, and Ping-Teng Chu wrote the manuscript. All authors read and approved the final manuscript.

\section{Acknowledgements}

The authors thank the enrollees of the National Health Insurance Research Database for their important contributions.

\section{References}

1. Bray F, Ferlay J, Soerjomataram I, Siegel RL, Torre LA, Jemal A. Global cancer statistics 2018 : GLOBOCAN estimates of incidence and mortality worldwide for 36 cancers in 185 countries. CA Cancer J Clin. 2018;68(6):394-424.

2. GLOBOCAN I. Estimated Cancer Incidence, Mortality and Prevalence Worldwide in 2012 v1. 0. IARC CancerBase No. 11. International Agency for Research on Cancer; 2013. 2012.

3. Vogel JD, Eskicioglu C, Weiser MR, Feingold DL, Steele SR. The American Society of Colon and Rectal Surgeons Clinical Practice Guidelines for the Treatment of Colon Cancer. Dis Colon Rectum. 2017;60(10):999-1017.

4. Chatterjee S, Khunti K, Davies MJ. Type 2 diabetes. The Lancet. 2017;389(10085):2239-51.

5. Zhou B, Lu Y, Hajifathalian K, Bentham J, Di Cesare M, Danaei G, et al. Worldwide trends in diabetes since 1980: a pooled analysis of 751 population-based studies with $4 \cdot 4$ million participants. The Lancet. 2016;387(10027):1513-30.

6. Holden SE. Diabetes and Cancer. Endocr Dev. 2016;31:135-45.

7. Larsson SC, Orsini N, Wolk A. Diabetes mellitus and risk of colorectal cancer: a meta-analysis. J Natl Cancer Inst. 2005;97(22):1679-87.

8. Force UPST. Screening for colorectal cancer: US Preventive Services Task Force recommendation statement. Ann Intern Med. 2008;149(9):627.

9. Mei ZB, Zhang ZJ, Liu CY, Liu Y, Cui A, Liang ZL, et al. Survival benefits of metformin for colorectal cancer patients with diabetes: a systematic review and meta-analysis. PLoS One. 2014;9(3):e91818.

10. Sehdev A, Shih YC, Vekhter B, Bissonnette MB, Olopade OI, Polite BN. Metformin for primary colorectal cancer prevention in patients with diabetes: a case-control study in a US population. Cancer. 2015;121(7):1071-8. 
11. Singh S, Singh H, Singh PP, Murad MH, Limburg PJ. Antidiabetic medications and the risk of colorectal cancer in patients with diabetes mellitus: a systematic review and meta-analysis. Cancer Epidemiol Biomarkers Prev. 2013;22(12):2258-68.

12. Zhang ZJ, Zheng ZJ, Kan H, Song Y, Cui W, Zhao G, et al. Reduced risk of colorectal cancer with metformin therapy in patients with type 2 diabetes: a meta-analysis. Diabetes Care. 2011;34(10):2323-8.

13. 13.

14. Fujita Y, Inagaki N. Metformin. New Preparations and Nonglycemic Benefits. Curr Diab Rep. 2017;17(1):5.

15. Paulus JK, Williams CD, Cossor FI, Kelley MJ, Martell RE. Metformin, Diabetes, and Survival among U.S. Veterans with Colorectal Cancer. Cancer Epidemiol Biomarkers Prev. 2016;25(10):1418-25.

16. Tsilidis KK, Kasimis JC, Lopez DS, Ntzani EE, loannidis JP. Type 2 diabetes and cancer: umbrella review of meta-analyses of observational studies. BMJ. 2015;350:g7607.

17. Zanders MM, Vissers PA, Haak HR, van de Poll-Franse LV. Colorectal cancer, diabetes and survival: epidemiological insights. Diabetes Metab. 2014;40(2):120-7.

18. 18.

19. Garcia-Perez LE, Alvarez M, Dilla T, Gil-Guillen V, Orozco-Beltran D. Adherence to therapies in patients with type 2 diabetes. Diabetes Ther. 2013;4(2):175-94.

20. 20.

21. Ma YS, Yang IP, Tsai HL, Huang CW, Juo SH, Wang JY. High glucose modulates antiproliferative effect and cytotoxicity of 5-fluorouracil in human colon cancer cells. DNA Cell Biol. 2014;33(2):6472.

22. 22.

23. Wilkinson JE, Culpepper L. Peer Reviewed:Associations Between Colorectal Cancer Screening and Glycemic Control in People With Diabetes, Boston, Massachusetts, 2005-2010. Preventing chronic disease. 2011;8(4).

24. Berster JM, Goke B. Type 2 diabetes mellitus as risk factor for colorectal cancer. Arch Physiol Biochem. 2008;114(1):84-98.

25. Jeon JY, Jeong DH, Park MG, Lee JW, Chu SH, Park JH, et al. Impact of diabetes on oncologic outcome of colorectal cancer patients: colon vs. rectal cancer. PLoS One. 2013;8(2):e55196.

26. Dehal AN, Newton CC, Jacobs EJ, Patel AV, Gapstur SM, Campbell PT. Impact of diabetes mellitus and insulin use on survival after colorectal cancer diagnosis: the Cancer Prevention Study-II Nutrition Cohort. J Clin Oncol. 2012;30(1):53-9.

27. Siddiqui AA, Spechler SJ, Huerta S, Dredar S, Little BB, Cryer B. Elevated HbA1c is an independent predictor of aggressive clinical behavior in patients with colorectal cancer: a case-control study. Dig Dis Sci. 2008;53(9):2486-94. 
28. Ahuja V, Chou CH. Novel Therapeutics for Diabetes: Uptake, Usage Trends, and Comparative Effectiveness. Curr Diab Rep. 2016;16(6):47.

29. Pollak M. Overcoming Drug Development Bottlenecks With Repurposing: Repurposing biguanides to target energy metabolism for cancer treatment. Nat Med. 2014;20(6):591-3.

30. Decensi A, Puntoni M, Goodwin P, Cazzaniga M, Gennari A, Bonanni B, et al. Metformin and cancer risk in diabetic patients: a systematic review and meta-analysis. Cancer Prev Res (Phila). 2010;3(11):1451-61.

31. Saraei P, Asadi I, Kakar MA, Moradi-Kor N. The beneficial effects of metformin on cancer prevention and therapy: a comprehensive review of recent advances. Cancer Manag Res. 2019;11:3295-313.

32. Coyle C, Cafferty FH, Vale C, Langley RE. Metformin as an adjuvant treatment for cancer: a systematic review and meta-analysis. Ann Oncol. 2016;27(12):2184-95.

33. Hosono K, Endo H, Takahashi H, Sugiyama M, Sakai E, Uchiyama T, et al. Metformin suppresses colorectal aberrant crypt foci in a short-term clinical trial. Cancer Prev Res (Phila). 2010;3(9):107783.

34. Spillane S, Bennett K, Sharp L, Barron TI. A cohort study of metformin exposure and survival in patients with stage I-III colorectal cancer. Cancer Epidemiol Biomarkers Prev. 2013;22(8):1364-73.

35. Garrett CR, Hassabo HM, Bhadkamkar NA, Wen S, Baladandayuthapani V, Kee BK, et al. Survival advantage observed with the use of metformin in patients with type II diabetes and colorectal cancer. Br J Cancer. 2012;106(8):1374-8.

36. Rattan R, Ali Fehmi R, Munkarah A. Metformin: an emerging new therapeutic option for targeting cancer stem cells and metastasis. J Oncol. 2012;2012:928127.

37. Tsai MJ, Yang CJ, Kung YT, Sheu CC, Shen YT, Chang PY, et al. Metformin decreases lung cancer risk in diabetic patients in a dose-dependent manner. Lung Cancer. 2014;86(2):137-43.

38. De Monte A, Brunetti D, Cattin L, Lavanda F, Naibo E, et al. Metformin and aspirin treatment could lead to an improved survival rate for Type 2 diabetic patients with stage II and III colorectal adenocarcinoma relative to non-diabetic patients. Molecular clinical oncology. 2018;8(3):504-12.

39. Fransgaard T, Thygesen LC, Gögenur I. Association between metformin use after surgery for colorectal cancer and oncological outcomes: A nationwide register- based study. International journal of cancer. 2018;143(1):63-72.

\section{Figures}


Patients with diagnosed colorectal cancer in the registry of catastrophic illness in 2000-2012 and had with curative surgery

$\mathrm{N}=91,649$

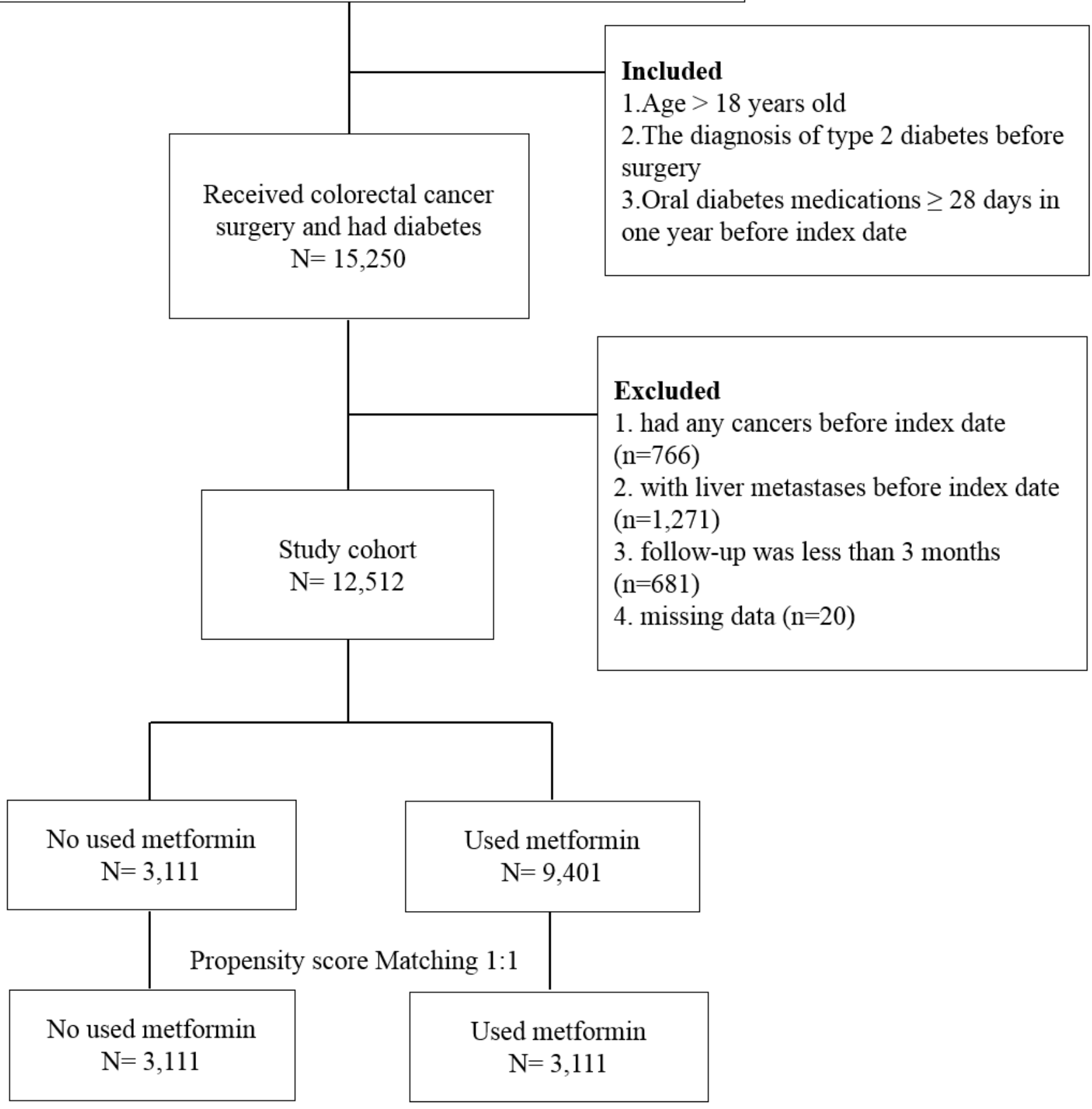

\section{Figure 1}

Flowchart of procedures to select original cohort and matched cohort for the study. 


\section{Matched cohort}

\section{Metformin using vs. non-using $\quad \mathrm{HR}(95 \% \mathrm{Cl}) \quad p$ value}

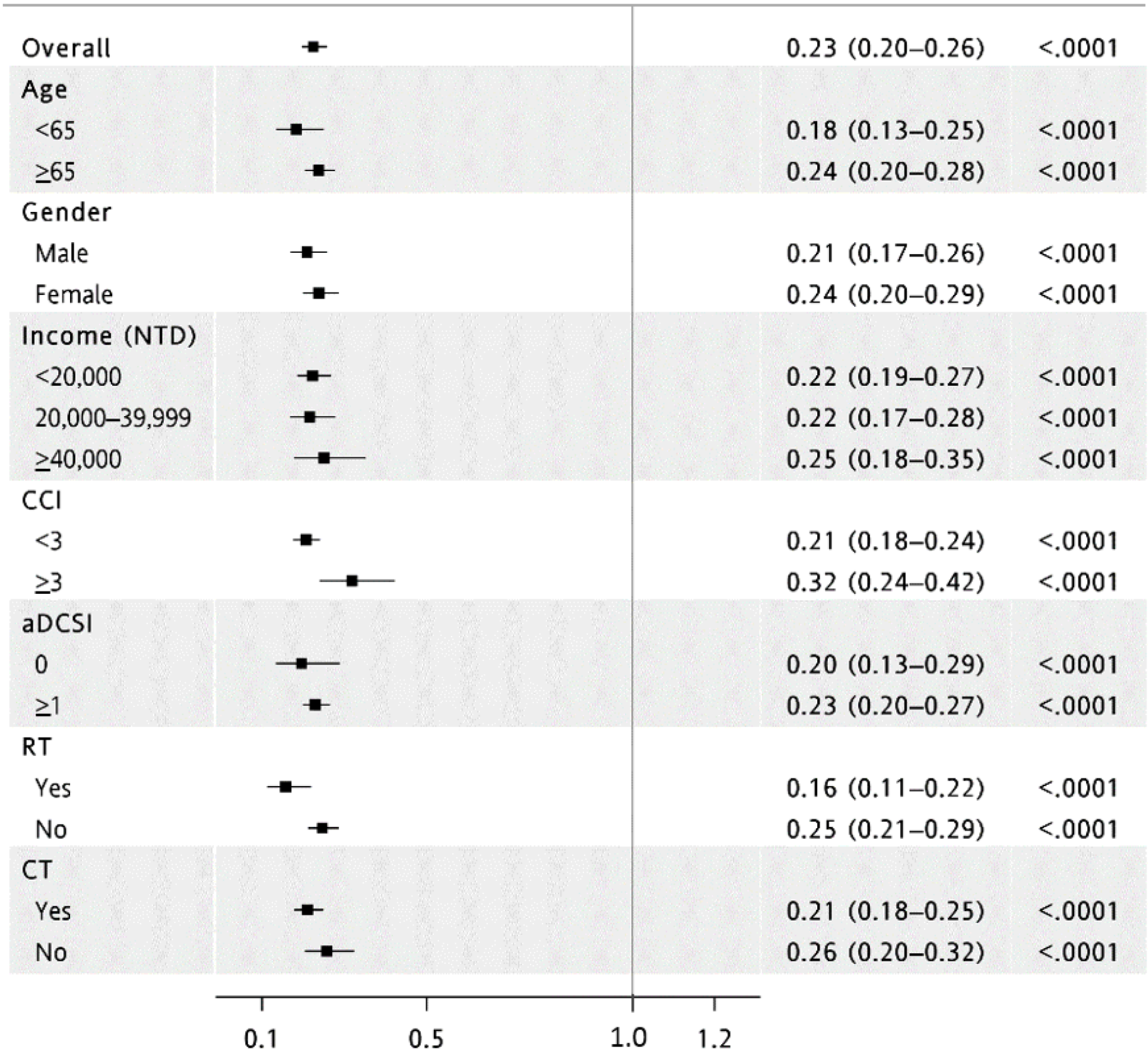

Figure 2

Cox proportional hazard analysis of 5-year survival in the matched cohorts. aDCSIXadapted Diabetes

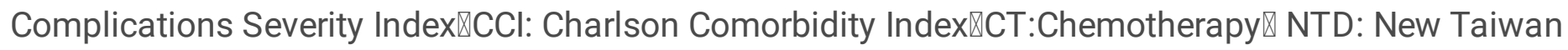
Dollars $₫ \mathrm{RT}$ : Radiotherapy $₫ S M D$, standardized mean difference. 


\section{Matched cohort}

\section{Metformin using vs. non-using $\quad \mathrm{HR}(95 \% \mathrm{Cl}) \quad p$ value}

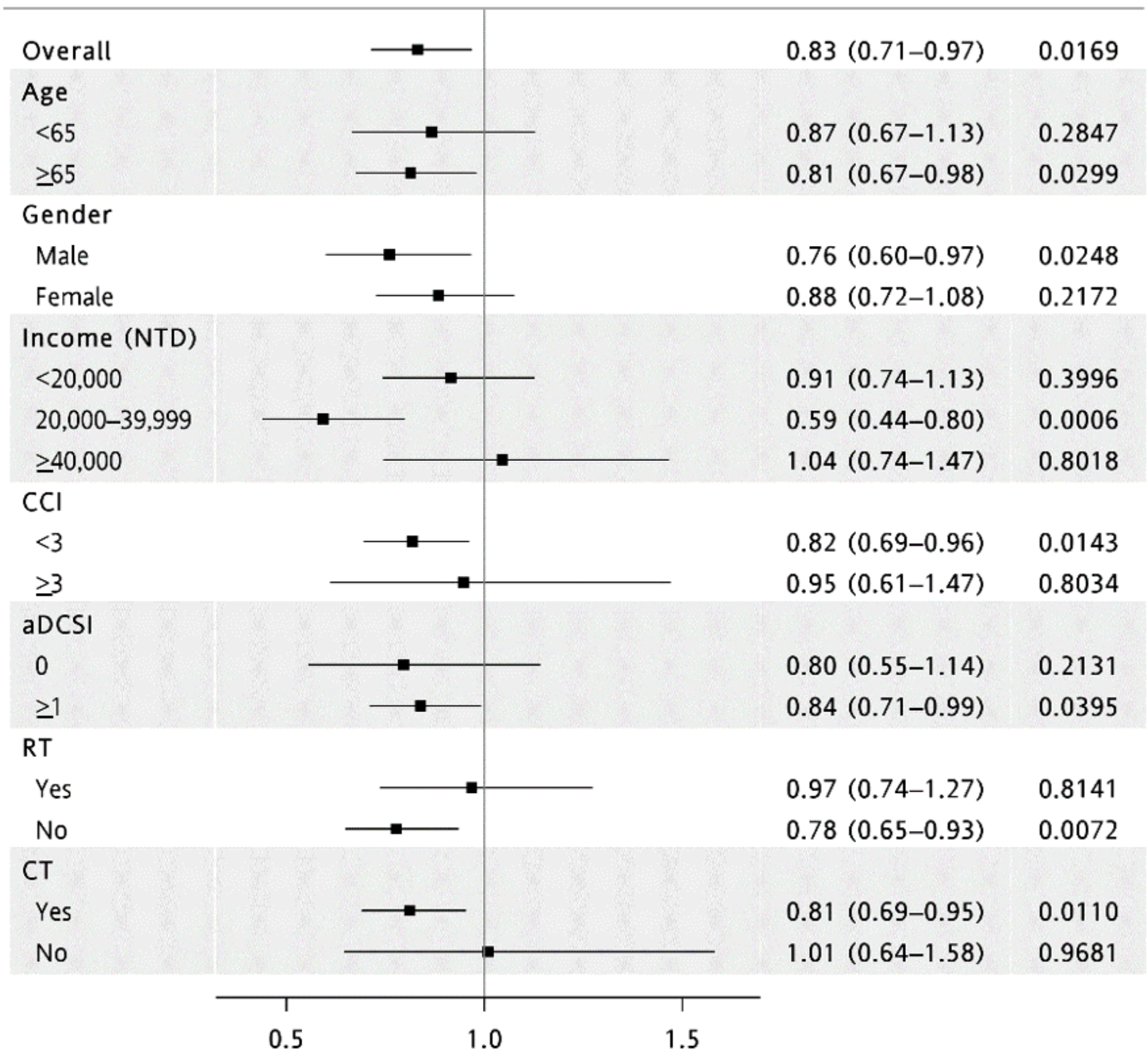

Figure 3

Cox proportional hazard analysis of liver metastasis in the matched cohorts. aDCSI囚adapted Diabetes

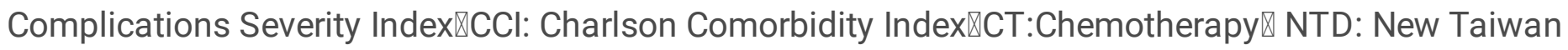

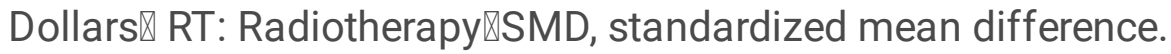




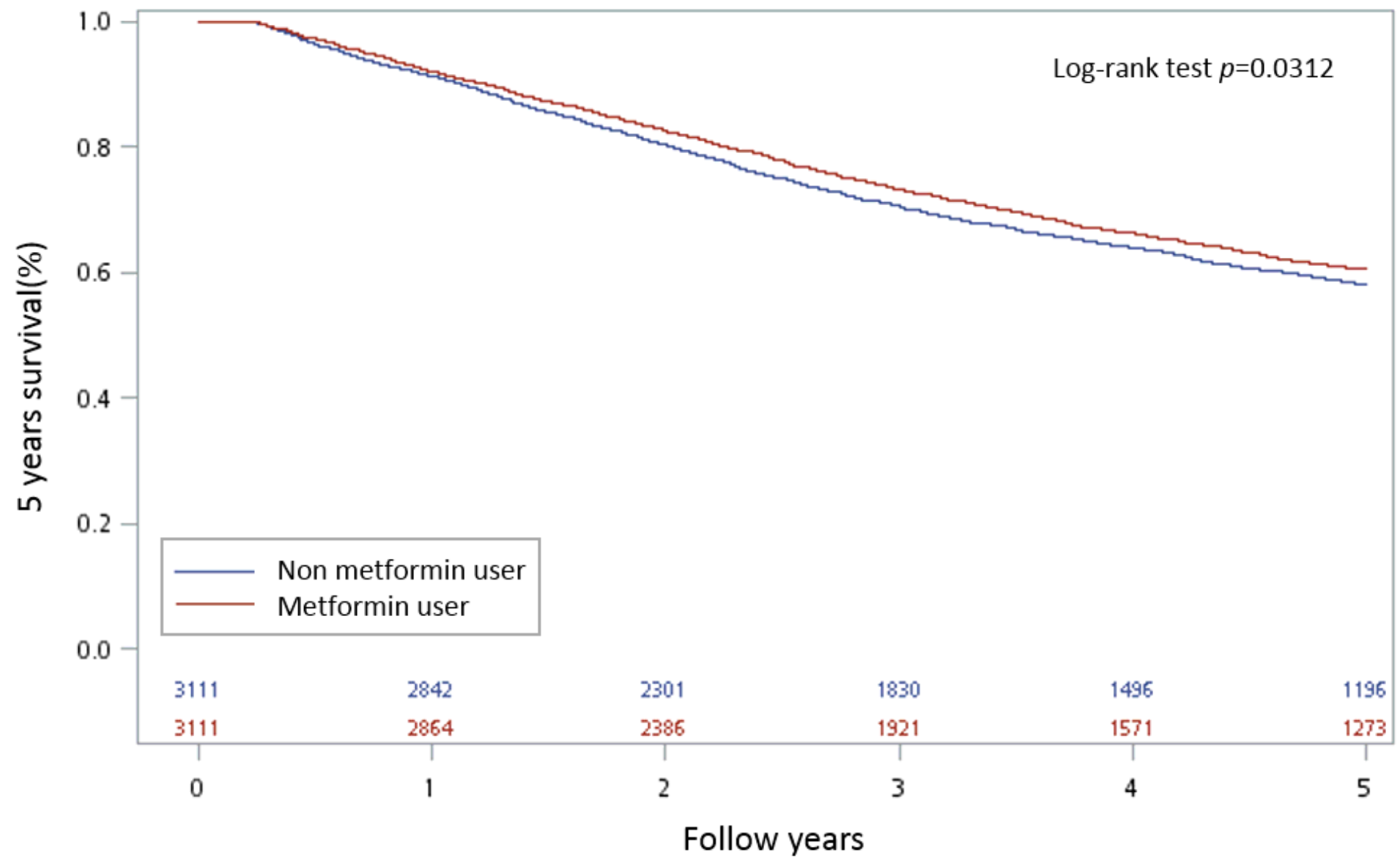

Figure 4

Kaplan Meyer curves of overall survival for 5-year 


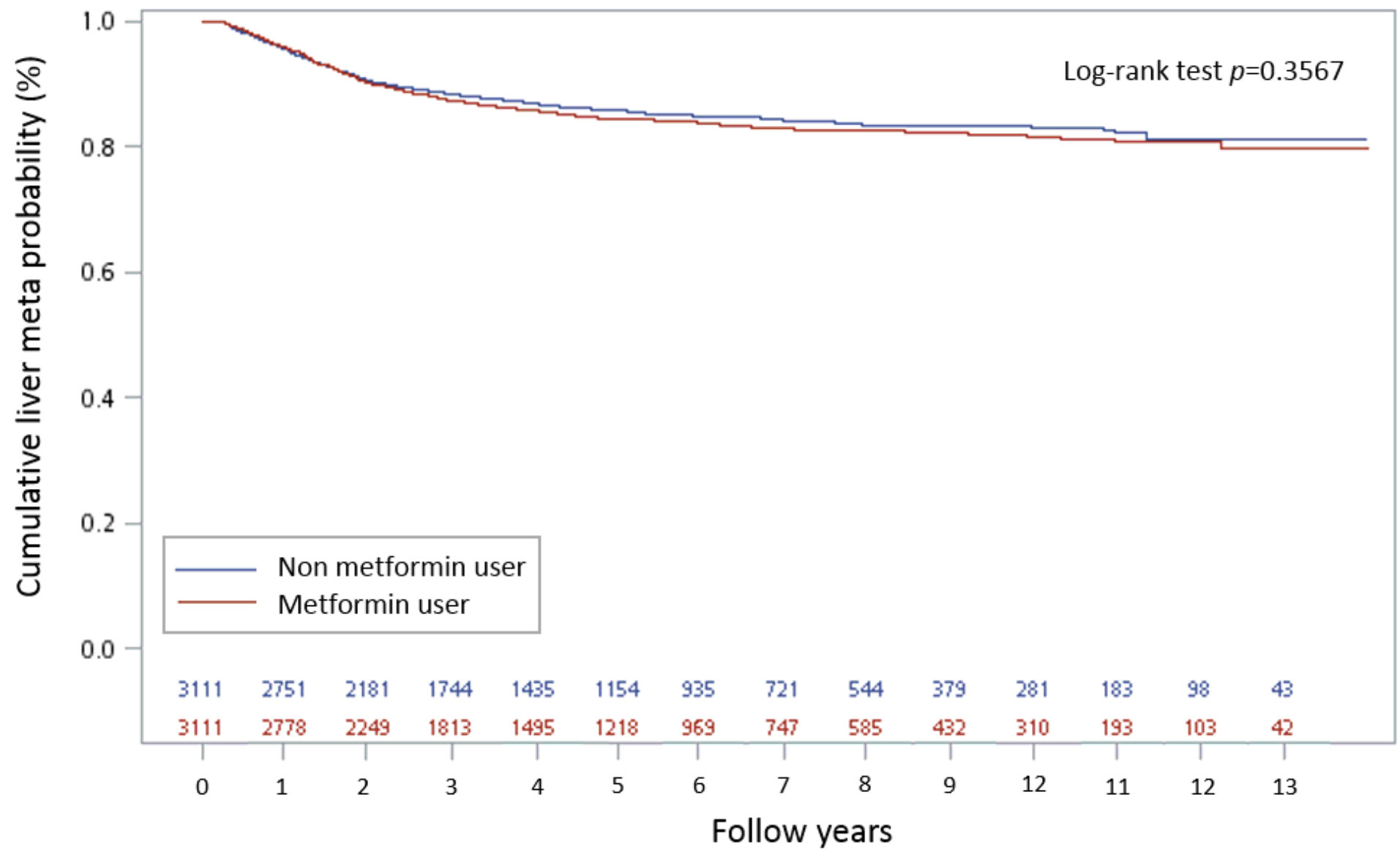

Figure 5

Kaplan Meyer curves of liver metastasis 